\title{
sciendo
}

\author{
BULGARIAN ACADEMY OF SCIENCES
}

CYBERNETICS AND INFORMATION TECHNOLOGIES • Volume 18, No 2

Sofia $2018 \quad$ Print ISSN: 1311-9702; Online ISSN: 1314-4081

DOI: $10.2478 /$ cait-2018-0032

\section{Evaluation of Two-Dimensional Angular Orientation of a Mobile Robot by a Modified Algorithm Based on Hough Transform}

\author{
Dmitry N. Aldoshkin, Roman Y. Tsarev \\ Department of Informatics, Siberian Federal University, Krasnoyarsk, Russia \\ E-mails: aldoshkind@gmail.com tsarev.sfu@mail.ru
}

\begin{abstract}
This paper proposes an algorithm that assesses the angular orientation of a mobile robot with respect to its referential position or a map of the surrounding space. In the framework of the suggested method, the orientation problem is converted to evaluating a dimensional rotation of the object that is abstracted as a polygon (or a closed polygonal chain). The method is based on Hough transform, which transforms the measurement space to a parametric space (in this case, a twodimensional space $[\boldsymbol{\theta}, r]$ of straight-line parameters). The Hough transform preserves the angles between the straight lines during rotation, translation, and isotropic scaling transformations. The problem of rotation assessment then becomes a one-dimensional optimization problem. The suggested algorithm inherits the Hough method's robustness to noise.
\end{abstract}

Keywords: Simultaneous Localization And Mapping (SLAM), path planning, Hough transform, angular orientation, mobile robot.

\section{Introduction}

To solve its functional tasks, a mobile robot must be able to orient in its surrounding space; that is, the robot must construct and follow its movement paths [1]. This path building in a space of known (partially or fully) geometrical configuration is presently solved in several ways [2]. Simultaneous Localization And Mapping (SLAM) methods map the unknown surrounding space and ensure that the movement follows the built path [3]. SLAM presupposes that the mobile robot gathers data on the geometry of the surrounding space and defines the shift vector and rotation angle of the robot between separate time points [4]. Mathematically, this problem becomes the problem of defining the parameters of similarity transformations between two samples of two-dimensional geometrical measurements. The first sample is a measurement of the surrounding space geometry taken by the mobile robot's sensors [5], and the second is a measurement of the space geometry in any arbitrary preceding moment of time, or the space map, which has been preset a priori [6]. One of the several solutions to this problem is presented by Iocchi and Nardi [7] and Großmann and Poli [8]. The algorithm is based on the Hough transform and 
notably inherits its robustness to measurement noise and outliers. Iocchi, Mastrantuono and Nardi [9] and Iocchi and Nardi [10] developed a probabilistic approach for solving the localization problem using the Hough transform, and Grisetti, Iocchi and Nardi [11] and Censi, Iocchi and Gri s e t t i [12] explored the applicability of this algorithm to global localization. The maps built by multiple robots have also been merged into a single map [13].

However, this algorithm has several drawbacks. First, its translation invariance is limited to small translation values; second, defining the rotation period demands computations in the measurement space. This paper proposes an algorithm that eliminates these disadvantages. The suggested algorithm defines the angle orientation at any value of translation, requires no computations in the measurement space, and derives all necessary information from the Hough accumulator alone.

\section{Problem setting}

Similarity transformations are transformations that combine rotation, translation, and isotropic scaling [14]. A point with coordinates $(x, y)$ transforms into a point with coordinates $\left(x^{\prime}, y^{\prime}\right)$ :

$$
\left[\begin{array}{l}
x^{\prime} \\
y^{\prime} \\
1
\end{array}\right]=\left[\begin{array}{ccc}
s \cos \alpha & s \sin \alpha & t_{x} \\
-s \sin \alpha & s \cos \alpha & t_{y} \\
0 & 0 & 1
\end{array}\right] \cdot\left[\begin{array}{l}
x \\
y \\
1
\end{array}\right],
$$

where $s$ is the scaling coefficient, $\alpha$ is the rotation angle, and $\left(t_{x}, t_{y}\right)^{\mathrm{T}}$ is the translation vector.

The transformation matrix calculated by (1) decomposes into a rotation matrix, a translation vector, and a scaling coefficient. Owing to this decomposition, the problem of assessing a similarity transformation can be divided into three subproblems: assessment of rotation, assessment of translation, and assessment of scaling.

In this paper, the relative angle orientation of the mobile robot is assessed by assessing the rotation angle $\alpha$ in the two-dimensional image of an object (a sample of the geometrical measurements of the surrounding space captured by sensors) relative to a reference image (analog sample or map). The suggested method imposes limitations on the types of objects suitable for transformation assessment. Specifically, the object must be described by a set of straight lines or segments (polygons in special cases). This limitation is imposed by the two-dimensional linear Hough transform on which the proposed method is based. Although this restriction narrows the application scope of the method, it admits a wide range of problems, primarily related to image analysis of man-made objects. The solvable problems include localization and mapping in man-made environments (navigation in rooms or urban environments) and the tracking of various man-made objects (specially marked automotive vehicles, buildings, and facilities) using aerial photography data. Furthermore, this limitation can be weakened or removed by approximating the object contours as straight lines or by generalizing the proposed method to curves with arbitrary parameterization [15]. 
As mentioned above, the proposed method is based on the Hough transform, which assesses the parameters of analytically predetermined curves using measurement samples. Hough patented his transform in 1962 [16] for estimating the parameters of straight lines from measurement samples. Several methods for estimating the parameters of parameterized arbitrary curves have since been developed [17]. In essence, the Hough transformation associates every measurement $m$ of a sample $M$, which has coordinates $(x, y)$ in the measurement space $[x, y]$ with a curve in the straight-line parameter space $[\theta, \rho]$ :

$$
\rho(\theta)=x \cos \theta+y \sin \theta .
$$

This equation describes a family (an infinite set) of lines in the measurement space passing through a point with coordinates $(x, y)$. However, as computer technology cannot handle infinite concepts, the space $[\theta, \rho]$ is discretized such that each point $(\theta, \rho)$ is associated with a value $(\theta, \rho)$, characterizing the number of points in sample $M$ on the line with parameters $(\theta, \rho)$. The elements $A(\theta, \rho)$ are assembled into a matrix $A$, called the accumulator matrix. $A$ is iteratively constructed by traversing all measurements $\mathrm{m}$ and plays a major role in further discourse. When the measurement points of the sample lie on distinct lines, the values of $A$ differ significantly. The parameters of straight lines that most closely fit the measurements of the original sample $M$ manifest as pronounced local maxima. However, the automatic selection of lines is beset by statistical challenges; especially, the quantification of local maxima is confounded by various noises derived from the source, sampling round-off, and other factors [18]. For this reason, direct determination of the maxima in matrix $A$ is not a suitable strategy for evaluating an object's image rotation.

The Hough transform preserves the angles between the lines. More specifically, it preserves the intervals on the $\theta$ axis of the accumulator between the maxima corresponding to the lines of the measurement space, regardless of the rotation angle of an object in the measurement space. Using this feature, one can evaluate the rotation angle without determining multiple maxima and their locations.

\section{Source data}

Similarity transformations are transformations that combine rotation, translation, and isotropic scaling [14]. Equation (1) transforms a point with coordinates $(x, y)$ into a point with coordinates $\left(x^{\prime}, y^{\prime}\right)$. The source data for the proposed algorithm are two samples of measurements of the surrounding space: a reference sample $M_{1}$ and a study sample $M_{2}$. To simplify the description without loss of generality, the samples are further regarded as binary images, i.e., two-dimensional matrices filled with zeros and ones: $I:(x, y) \rightarrow\{0,1\}, x=\overline{0, w} ; y=\overline{0, h}$, where $w$ and $h$ are the image width and height, respectively. Thus, the images of samples $M_{1}$ and $M_{2}$ are denoted as $I_{1}$ and $I_{2}$, respectively. In the subsequent reasoning, we assume identical sizes of the sample images; that is, $w\left(I_{1}\right)=w\left(I_{2}\right), h\left(I_{1}\right)=h\left(I_{2}\right)$, and determine the rotation angle between the two synthetic (model) samples $M_{A}$ and $M_{B}$. Here sample $M_{A}$ imitates a space map and, $M_{B}$ imitates noisy measurements of this space. The sample images are illustrated in Fig. 1. 


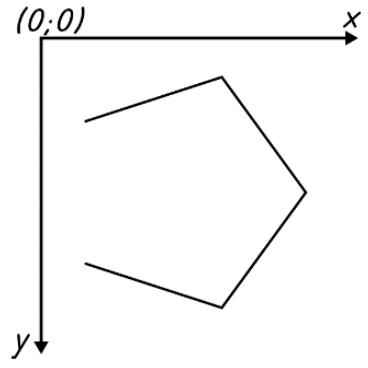

(a)

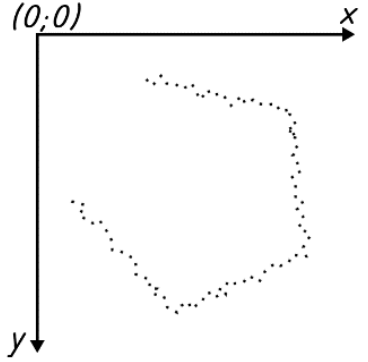

(b)

Fig. 1. Images of model samples of measurements of the surrounding space: (a) sample $M_{A}$ (map); (b) sample $M_{B}$ (measurements with noise)

\section{Concept of the method}

The proposed algorithm is based on the following concept: If a set of lines in twodimensional space rotates through the same angle, the angular coefficients of all lines in the set vary by the same value (the angle of rotation). Therefore, the difference between the angular coefficients of any two lines remains unchanged.

In the corresponding space of the Hough accumulator $A$, the maxima corresponding to the lines of the image space will be located at a constant distance from each other along the $\theta$ axis, regardless of the rotation angle of the image. Meanwhile, a rotation in the image space corresponds to a translation along the $\theta$ axis in the accumulator space $A$.

To determine the rotation angle, we should unambiguously identify the correspondence between the maxima $A_{1}$ and $A_{2}$ and then calculate their relative translation along the $\theta$ axis. The relative translation corresponds to the rotation angle $\alpha$ in the image space.

However, correlating the maxima is complicated owing to various noises, possible incompleteness of the information, different number of measurements per length unit of the line, and ambiguity in choosing the pair of maxima for angle determination. To ensure a stable solution, the proposed algorithm builds distributions $D\left(A_{1}, \theta\right)$ and $D\left(A_{2}, \theta\right)$ of the maxima (respectively referred to as $D_{1}(\theta)$ and $D_{2}(\theta)$ for brevity) along the $\theta$ axis and then computes the rotation angle $\alpha$ as a translation of distribution $D_{2}(\theta)$ relative to $D_{1}(\theta)$ at the maximum of some component of a function. The comparing function is given by

$$
\operatorname{cmp}\left(D_{1}(\theta), D_{2}(\theta+\alpha)\right) \underset{\alpha}{\rightarrow} \max .
$$

In the proposed algorithm, the comparing function is a correlation function.

\section{Constructing the accumulator of the Hough transform}

The first step of the proposed algorithm constructs the accumulator $A$ of the Hough transform by the following moves:

1. Choose the size and resolution of both axes of the accumulator;

2. Fill the accumulator cells. 
Clearly, the size of the accumulator along the $\theta$ axis is $\left|A_{\theta}\right|=2 \pi$. The resolution of the accumulator along the $\theta$ axis (denoted as $\left\langle A_{\theta}\right\rangle$ ) defines the computational accuracy of the rotation angle, which should be as high as possible. However, for each $(w \times h)$-sized image $I$, there is a limit $\left\langle A_{\theta}\right\rangle_{\max }$, which, when exceeded, will not increase the accuracy. $\left\langle A_{\theta}\right\rangle_{\max }$ is calculated as follows:

$$
\left\langle A_{\theta}\right\rangle_{\max }=\frac{2 \pi}{\theta_{I \lim }},
$$

where $\theta_{\text {lim }}$ is the limiting angle of line legibility in image $I$.

Below $\theta_{\text {Ilim }}$, any three points of the image cannot unambiguously belong to two lines because the discreteness of $I$ might erroneously assign two points to the same line and the third to a different line. $\theta_{\text {lim }}$ is determined by points along oppositely oriented lines in $I$ and is calculated as follows:

$$
\theta_{I \lim }=\operatorname{arctg}\left(\frac{b}{b^{2}+a \cdot(a-1)}\right)
$$

where $a=\max (w, h)$ and $b=\min (w, h)$.

Inserting (5) into (4), we obtain $\left\langle A_{\theta}\right\rangle_{\max }$ as the function of the size of $I$.

From personal experience, it is recommended that the selected $\left\langle A_{\theta}\right\rangle$ satisfies $\max (w, h) \leq\left\langle A_{\theta}\right\rangle<\left\langle A_{\theta}\right\rangle_{\max }$.

The size of the accumulator along the $\rho\left(\left|A_{\rho}\right|\right)$ axis is limited by the size $(w, h)$ of the image $I$, which fully contains the radius vector of every line in the image. Therefore, the length of this axis cannot exceed the diagonal of $I$, calculated by

$$
d(I)=\sqrt{\left(w(I)^{2}+h(I)^{2}\right)} .
$$

Assuming that $\left|A_{\rho}\right|=d(I)$, the range of the $\rho$ axis is $\rho \in[0, d(I)]$.

However, when constructing the accumulator with $\left|A_{\rho}\right|=d(I)$ in a coordinate system with an arbitrary chosen origin, the whole sample can be placed only in a single quarter of the coordinate plane. In later steps of the proposed algorithm, this leads to failed distribution of the peaks in the accumulator; consequently, the rotation angle is incompliant with (3). To solve this problem, we describe any line as two coincident lines with parameters $(\theta, \rho)$ and $(\theta+\pi,-\rho)$. In this approach, the $\rho$ axis ranges as $\rho \in[-d(I), d(I)]$, and $\left|A_{\rho}\right|$ is

$$
\left|A_{\rho}\right|=2 d(I) \text {. }
$$

This approach eliminates the problem of choosing the origin of the coordinate system but introduces the problem of choosing the rotation direction. A solution to the rotation problem will be discussed later.

The accumulator cells are filled by sequentially computing the values of each cell:

$$
\begin{aligned}
& A(\theta, \rho)=\sum_{i=0}^{w(I)} \sum_{j=0}^{h(I)}\left(f-\left(\rho-\left(x_{i} \cos \theta+y_{j} \sin \theta\right)\right) \cdot I(i, j),\right. \\
& \theta=\overline{0,\left|A_{\theta}\right|, \rho=\overline{0,|A \rho|},}
\end{aligned}
$$

where

$$
f(x)= \begin{cases}1, & x=0, \\ 0, & x \neq 0 .\end{cases}
$$

Fig. 2 shows an example of the accumulators constructed by this approach. 


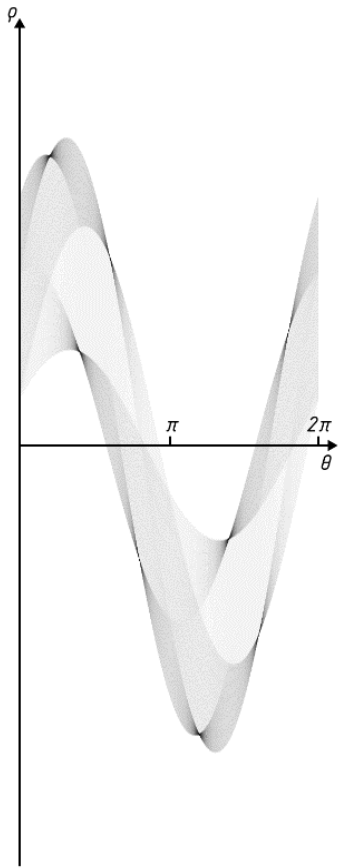

(a)

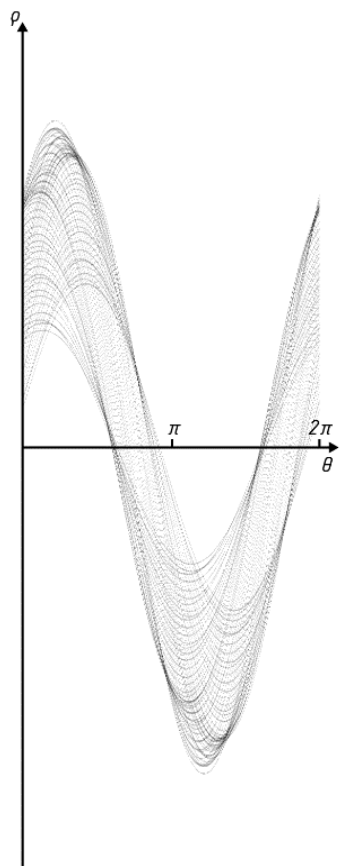

(b)

Fig. 2. Accumulators of the sample $M_{A}$ (a) and sample $M_{B}(\mathrm{~b})$

\section{Constructing an extreme case of the accumulator along the $\theta$ axis}

To construct an extreme distribution of maxima in the accumulator matrix, we select the maximum value of the accumulator at each value of the angle $\theta$ :

(10) $D(A, \theta)=\max (A(\theta, \rho)), \rho=\overline{-d(I), d(I)}$.

The distributions of the maxima calculated by (10) are displayed in Fig. 3.

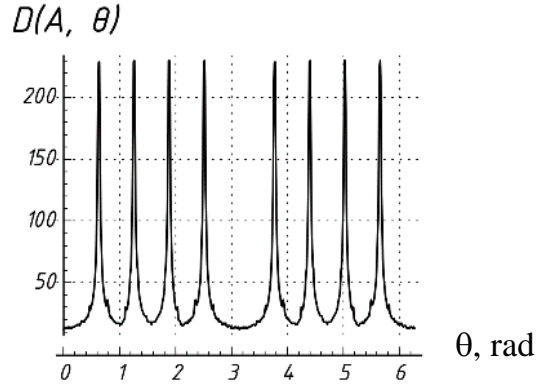

(a)

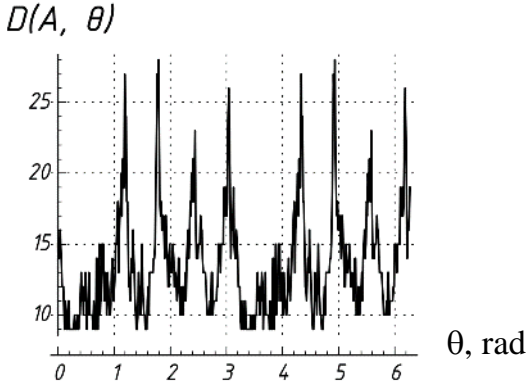

(b)

Fig. 3. Distributions of maxima in: (a) the accumulator of sample $M_{A}$; (b) the accumulator of sample $M_{B}$ 


\section{Calculation of the rotation angle}

As mentioned earlier, the rotation angle $\alpha$ is calculated as the relative translation between two maxima distributions, where some component of the function is maximized, and the comparing function is a correlation function. Thus, by substituting an arbitrary comparison function with the correlation function in (3), we can determine the rotation angle $\alpha$ by

$$
\operatorname{cor}\left(D_{1}(\theta), D_{2}(\theta+\alpha)\right) \underset{\alpha}{\rightarrow} \max , \quad \alpha=\overline{0,2 \pi}
$$

Finally, the correlation function is calculated as follows:

$$
\operatorname{cor}(X, Y)=\frac{\sum_{i=0}^{|X||Y|}\left(x_{i}-M[X]\right) \cdot\left(y_{i}-M[Y]\right)}{\sqrt{\sum_{i=0}^{X \mid}\left(x_{i}-M[X]\right)^{2}} \sqrt{\sum_{i=0}^{|Y|}\left(y_{i}-M[Y]\right)^{2}}} .
$$

Applying the correlation function (12) to the pair of distributions derived from (10), we obtain a curve with clearly pronounced maxima, whose main defines the rotation angle $\alpha$ through (11) (Fig. 4).

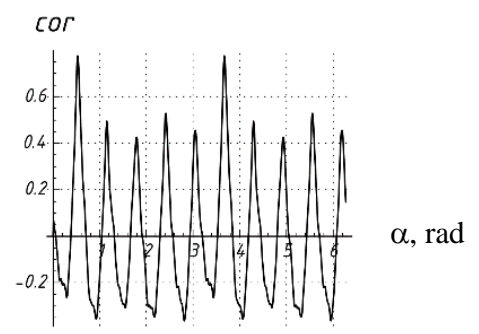

Fig. 4. Result of applying the correlation function to the distributions of Fig. 3

Fig. 4 displays 10 maxima corresponding to 10 values of the rotation angle. Five of these maxima correspond to correct rotation angles; the remaining false maxima (which generally comprise half the total number of maxima) manifest from duplications of the straight-line descriptions and doubling of the accumulator size along the $\rho$ axis (as stipulated earlier). At this stage, two solutions $(\alpha$ and $\alpha+\pi)$ achieve equal maxima of the comparing function. The correct value is determined by additional computations.

\section{Calculation of the rotation direction}

The rotation angle calculation yields a pair of values $(\alpha$ and $\alpha+\pi)$. The correct member of this pair cannot be determined from the maxima distribution alone.

As evident from Fig. 2, each column $A(\theta)$ of the accumulator $A$ contains nonzero values within a certain range (here denoted as $[l(A, \theta), u(A, \theta)]$. To solve the abovementioned problem of finding the rotation direction, we construct a second accumulator with each point $(\theta, \rho)$ replaced by its corresponding point $(\theta+\pi,-\rho)$. Therefore, if the range of $\theta$ is $[u(A, \theta), l(A, \theta)]$, the range of $\theta+\pi$ is $[l(A, \theta+\pi)=$ $=-u(A, \theta), u(A, \theta+\pi)=-l(A, \theta)]$. This follows from the principle of construction of the accumulator (Fig. 5). 
The lower bound $l(A, \theta)$ of column $A(\theta)$ is selected as the minimum value of $\rho$, for which $A(\theta, \rho)$ differs from zero:

$$
l(A, \theta)=\min (\rho), A(\theta, \rho)>0, \rho=\overline{0,\left|A_{\rho}\right|} .
$$

Similarly, the upper bound $u(A, \theta)$ of column $A(\theta)$ is chosen as the maximum value of $\rho$, for which $A(\theta, \rho)$ differs from zero:

$$
u(A, \theta)=\max (\rho), A(\theta, \rho)>0, \rho=\overline{0,\left|A_{\rho}\right|} .
$$

The values in columns $A(\theta)$ and $A(\theta+\pi)$ are identical but oppositely ordered along the $\rho$ axis (Fig. 6).

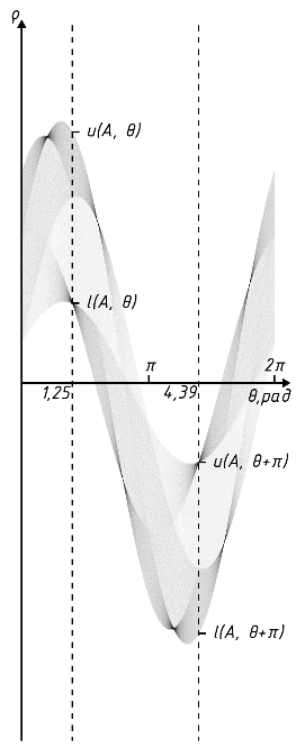

Fig. 5. Ranges of non-zero values in the columns of the accumulator matrix for $\theta=1.25$

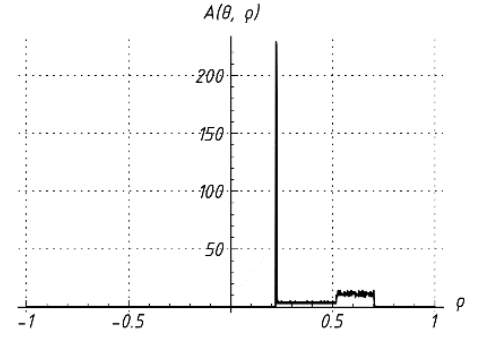

(a)

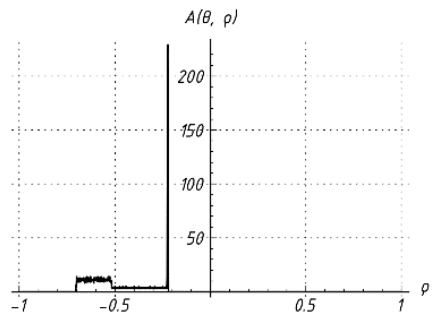

(b)

Fig. 6. Distribution of values in the columns of the accumulator matrix of Fig. 5 for: (a) $A(\theta=1.25)$; (b) $A(\theta=4.39)$

The range width of the non-zero values in a column is defined as the difference between the upper and lower bounds:

$$
w(A, \theta)=u(A, \theta)-l(A, \theta) .
$$

The middle of the range is obviously calculated by summing the lower bound and half of the range width: 


$$
m(A, \theta)=l(A, \theta)+0.5 \cdot w(A, \theta) .
$$

In general, the distribution of values in the range $[l(A, \theta), u(A, \theta)]$ for any $\theta$ is asymmetric about the center. The asymmetry of the values in a column is computed as the weighted sum of non-zero values in the column. Here, the weights are proportional to the translation (i.e., the distance between the current value and the mid-range):

$$
s(A, \theta)=\sum_{\rho=l(A, \theta)}^{u(A, \theta)} \frac{(\rho-m(A, \theta))}{(w(A, \theta))} \cdot A(\theta, \rho) .
$$

From the calculated $s(A, \theta)$ at each $\theta$, an asymmetry map $S(A)$ is obtained for the given accumulator (Fig. 7).

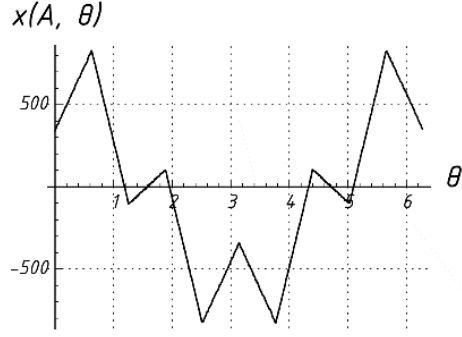

(a)

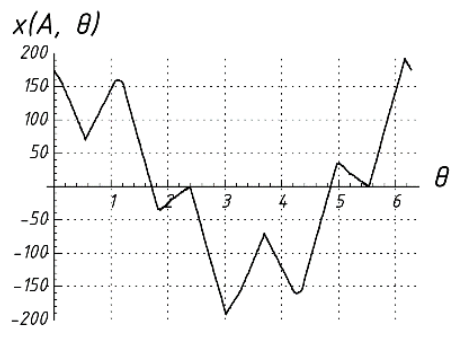

(b)

Fig. 7. Constructed asymmetry maps for accumulators of: (a) sample $M_{A}$; (b) sample $M_{B}$

As the asymmetry is determined by the geometry of the surrounding space, the accumulators of the two initial samples will yield similar asymmetry maps. Selecting the correct rotation angle from $\alpha$ and $\alpha+\pi$ reduces to choosing the angle at which the comparing function (18) is maximized in both asymmetry maps (Fig. 8):

$$
\operatorname{cor}\left(S\left(A_{1}\right), S\left(A_{2}\right)\right) \underset{\alpha}{\rightarrow} \max , \quad \alpha=\{\alpha, \alpha+\pi\} \text {. }
$$

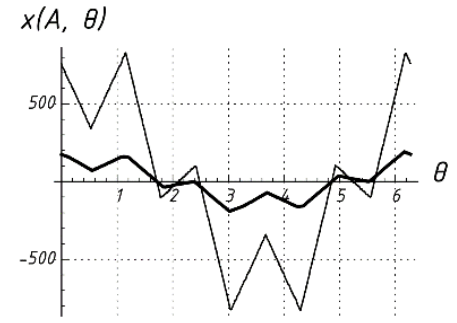

(a)

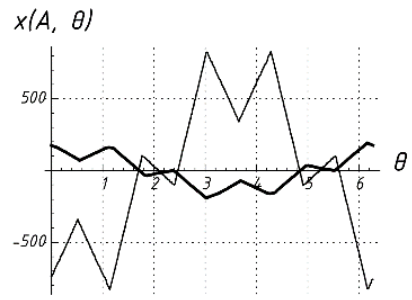

(b)

Fig. 8. Asymmetry maps shown in Fig. 7, with relative translations of: (a) $\alpha=0.52$; (b) $\alpha+\pi=3.66$

\section{Special cases}

In certain scene configurations, the rotation angle of the mobile robot cannot be found by the proposed method. For instance, the correct angle among $\mathrm{n}$ rotation angles cannot be decided in symmetric scenes (where $n$ is the order of symmetry of the scene) because the comparing function yields $2 n$ maxima. In the idealized case, these 
maxima are indistinguishable and the angle will be selected at random. In non-ideal cases, the maxima will differ under noise in the measurements and distortions incurred by discretization. In this case, the selected angle is affected by random factors and is generally incorrect.

When all straight lines are parallel (a special case of a symmetric scene), the rotation angle cannot be determined and the symmetry map loses its informativeness; that is, all values are zero in the ideal case. In real calculations, the values will specify the amounts of various noises.

Determining the rotation angle in such configurations requires additional information of the surrounding environment (non-geometric parameters of objects in the scene, such as color) or information that is independent of the surrounding space (such as inertial navigation data or odometry).

\section{Conclusion}

This paper considers the angular orientation of a mobile robot in its surrounding environment, a topical theme in SLAM frameworks. The proposed algorithm for determining the angular orientation is based on a two-dimensional linear Hough transform and exploits its remarkable property, namely that the relative positions of the accumulator maxima along the $\theta$ axis are invariant to rotation. Meanwhile, the translation of the maxima distribution corresponds to the rotation angle.

The source data for the algorithm are two samples of measurements of the surrounding space geometry, which have been received by the mobile robot's sensors. Thus, the problem of determining the angular orientation becomes the problem of determining the rotation angle of one sample relative to the other. In the proposed algorithm, the latter problem is reduced to defining the mutual translation of the distributions of the maxima in the accumulators built from the input samples. This translation is iteratively defined by displacing one distribution relative to another distribution containing the maximum (minimum) of some comparing function (in the present case, the comparing function is a correlation function). The resulting translation values correspond to the rotation angle.

The algorithm inherits the robustness of the Hough transform to measurement noise and outliers. Furthermore, the algorithm is tolerant to translation and isotropic scaling of sets. By virtue of these features, no preprocessing of the samples is required.

The algorithm has several limitations; especially, the geometry of the surrounding space should be dominated by straight lines (because we have chosen the linear Hough transform) and the rotation angle cannot be computed in symmetric scenes. The first limitation can be overcome by Hough transforms with different parameterization or by approximating the geometry with straight lines. To resolve the second limitation, we propose the use of additional (non-geometric) information on the surrounding environment.

Acknowledgements: The authors are thankful to Enago (www.enago.com) for the review of the English language of the paper. 


\section{References}

1. Y i, Y., X. H u. Robot Simultaneous Localization and Mapping Based on Self-Detected Waypoint. - Cybernetics and Information Technologies, Vol. 16, 2016, No 2, pp. 212-221.

2. Aldoshkin, D., A. Pupkov, R. Ts are v. Fast Algorithm of Planning a Robot's Path in 2-Dimensional Space on the Basis of Triangulation. - In: Proc. of 16th International Multidisciplinary Scientific Geoconference, Bulgaria, 2016, pp. 219-230.

3. Durrant-Whyte, H., T. B a i le y. Simultaneous Localisation and Mapping (SLAM): Part I. The Essential Algorithms. - IEEE Robotics \& Automation Magazine, Vol. 13, 2006, No 2, pp. 99-110.

4. Yi, Y., Y. Hu an g. Landmark Sequence Data Association for Simultaneous Localization and Mapping of Robots. - Cybernetics and Information Technologies, Vol. 14, 2014, No 3, pp. 212-221.

5. Ca i, J., X. Ru a n, P. Li. Autonomous Path Planning Scheme Research for Mobile Robot. Cybernetics and Information Technologies, Vol. 16, 2016, No 4, pp. 212-221.

6. P e n g, J., Y. Hu an g, G. Lu o. Robot Path Planning Based on Improved A* Algorithm. Cybernetics and Information Technologies, Vol. 15, 2015, No 2, pp. 171-180.

7. I o c chi, L., D. Nardi. Self-Localization in the RoboCup Environment. - In: Proc. of 3rd International Workshop on RoboCup, Sweden, 1999, pp. 318-330.

8. Gro $\beta \mathrm{m}$ a n n, A., R. P o l i. Robust Mobile Robot Localisation from Sparse and Noisy Proximity Readings Using Hough Transform and Probability Grids. - Robotics and Autonomous Systems, Vol. 37, 2001, No 1, pp. 1-18.

9. I o c c h i, L., D. M a s tr a n t u o n o, D. N a r d i. A Probabilistic Approach to Hough Localization. - In: Proc. of IEEE International Conference on Robotics and Automation, Korea, 2001, pp. 4250-4255.

10. I o c c h i, L., D. N a rdi. Hough Localization for Mobile Robots in Polygonal Environments. Robotics and Autonomous Systems, Vol. 40, 2002, No 1, pp. 43-58.

11. Grisetti, G., L. Ioc chi, D. Nardi. Global Hough Localization for Mobile Robots in Polygonal Environments. - In: Proc. of IEEE International Conference on Robotics and Automation, US, 2002, pp. 353-358.

12. Ce n s i, A., L. I o c c h i, G. G r i s e t t i. Scan Matching in the Hough Domain. - In: Proc. of IEEE International Conference on Robotics and Automation, Spain, 2005, pp. 2739-2744.

13. S a e edi, S., L. P a u 11, M. Trentini, M. S e to, H. Li. Map Merging for Multiple Robots Using Hough Peak Matching. - Robotics and Autonomous Systems, Vol. 62, 2014, No 10, pp. 1408-1424.

14. Hartley, R., A. Zi s s e rman. Multiple View Geometry in Computer Vision. Cambridge: Cambridge University Press, 2003.

15. B a 11 ard, D. H. Generalizing the Hough Transform to Detect Arbitrary Shapes. - Pattern Recognition, Vol. 13, 1981, No 2, pp. 111-122.

16. Hough, V. C. P. Patent US3069654 A, G 06 K 9/46, G 01 T 5/02. Method and Means for Recognizing Complex Patterns. Applicant and Patent Holder Paul V. C. Hough. No 19600325; Application. 25.03.60; Publ. 18.12.62.

17. D u d a, R. O., P. E. H a r t. Use of the Hough Transformation to Detect Lines and Curves in Pictures. - Communications of the ACM, Vol. 15, 1972, No 1, pp. 11-15.

18. C h e n, W., X. W u, Y. L u. An Improved Path Planning Method Based on Artificial Potential Field for a Mobile Robot. - Cybernetics and Information Technologies, Vol. 15, 2015, No 2, pp. 181-191. 